\title{
Defective ORF8 dimerization in delta variant of SARS CoV2 leads to abrogation of ORF8 MHC-I interaction and overcome suppression of adaptive immune response
}

\author{
Armi M Chaudhari ${ }^{1 \square}$, Indra Singh ${ }^{1} \square$, Madhvi Joshi ${ }^{1}$, Amrutlal Patel ${ }^{1}$, and Chaitanya \\ Joshi $^{1 *}$ \\ ${ }^{1}$ Gujarat Biotechnology Research Centre (GBRC), Department of Science and Technology, \\ Government of Gujarat, Gandhinagar-382011
}

$\square$ Equal Contribution: Authors had contributed equally.

*Corresponding author: Chaitanya Joshi, Gujarat Biotechnology Research Centre (GBRC), Department of Science and Technology, Government of Gujarat, 6th Floor, Block B\&D, MS Building, Gandhinagar-382011. Tel/Fax: 079-232-58680; E-mail: director@gbrc.res.in

\section{Abstract}

In India, the breakthrough infections during second wave of COVID-19 pandemic was due to SARS-COV-2 delta variant (B.1.617.2). It was reported that majority of the infections were caused by the delta variant and only $9.8 \%$ percent cases required hospitalization whereas, only $0.4 \%$ fatality was observed. Sudden dropdown in COVID-19 infections was observed within a short timeframe, suggesting better host adaptation with evolved delta variant. Down regulation of host immune response against SARS-CoV-2 by ORF8 induced MHC-I degradation has been reported earlier. The Delta variant carried mutations (deletion) at Asp119 and Phe120 amino acids which are critical for ORF8 dimerization. The deletions of amino acids Asp119 and Phe120 in ORF8 of delta variant results in structural instability of ORF8 dimer caused by disruption of hydrogen bonding and salt bridges as revealed by structural analysis and MD simulation studies of ORF8 dimer. Further, flexible docking of wild type and mutant ORF8 dimer revealed reduced interaction of mutant ORF8 dimer with MHC-I as compared to wild type ORF8 dimer with MHC-1, thus implicating its possible role in MHC-I expression and host immune response against SARS-CoV-2. We thus propose that mutant ORF8 may not hindering the MHC-I expression thereby resulting in better immune response against SARS-CoV-2 delta variant, which partly explains the sudden drop of SARS-CoV-2 infection rate in the second wave of SARS-CoV-2 predominated by delta variant in India

Key words: SARS-COV-2, Delta variant, COVID-19, MHC1, ORF8, Protein dimerization, Protein-Protein interactions, MD simulations. 

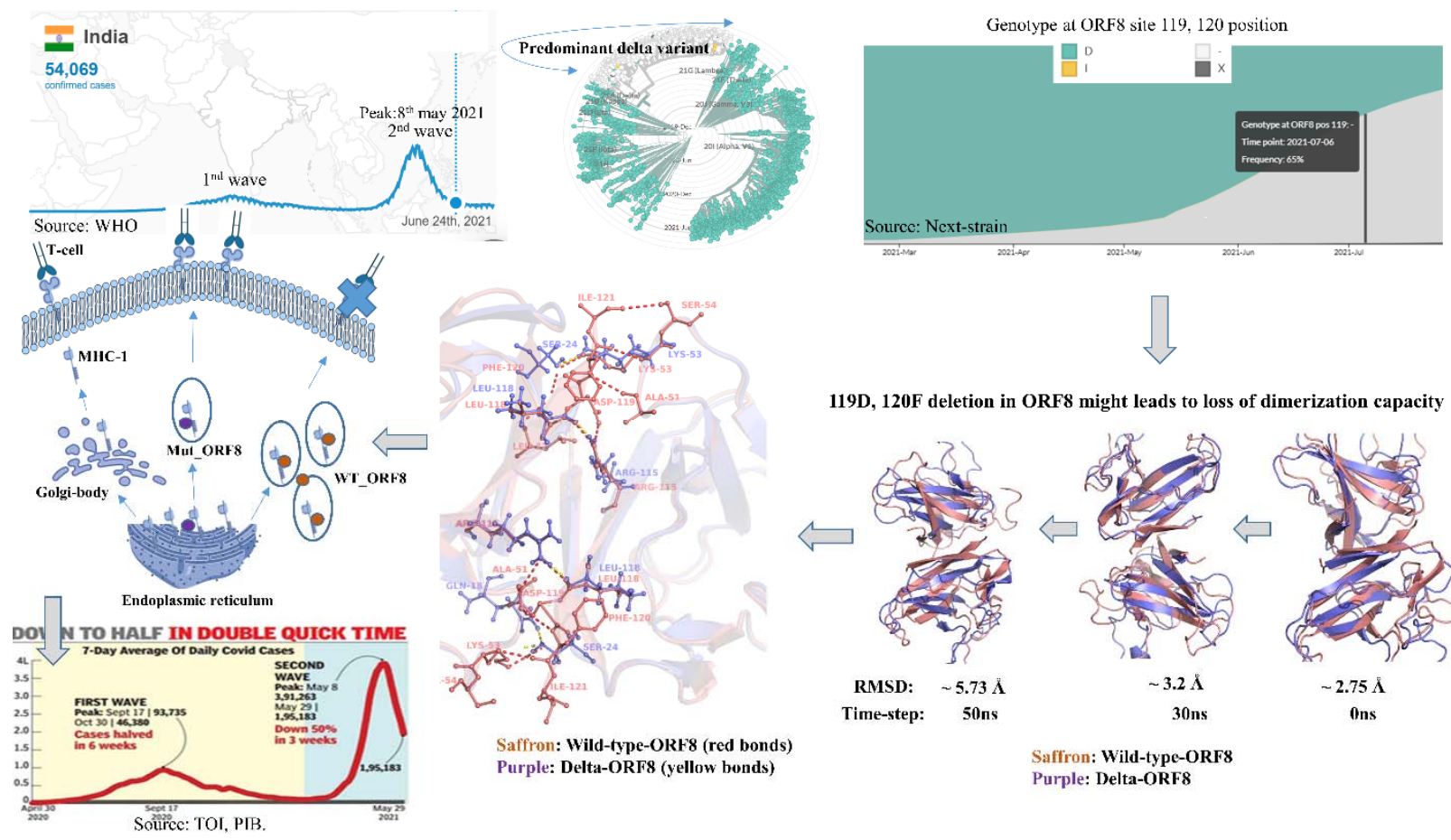

119D, 120F deletion in ORF8 might leads to loss of dimerization capacity
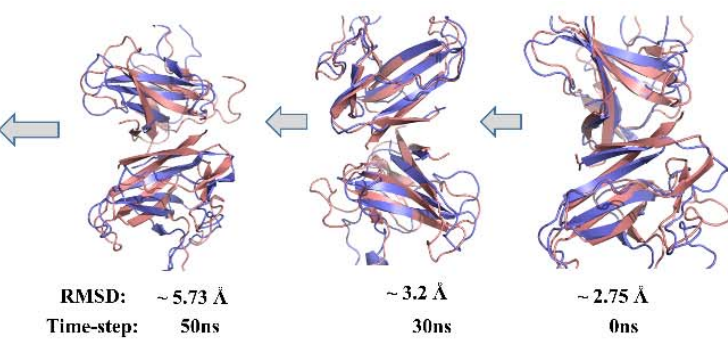

Saffron: Wild-type-ORF8

Purple: Delta-ORF8

\section{Introduction}

SARS-CoV-2 pandemic had infected more than 199 million people and more than 4 million deaths worldwide till $4^{\text {th }}$ August 2021. During this pandemic, virus had mutated to evade the host immune system and also to enhance its transmission. These variants were detected using high throughput sequencing methods and their effect on virus is studied extensively. With these evolving variants, SARS-CoV-2 Interagency Group (SIG) of US government come up with Variant Classification scheme that defines three classes of SARS-CoV-2 variants, such as 1) VOI 2) VOC and 3) VOHC. Among them, delta variant belonging to the group of VOCs had surged to sudden increases in infection during second wave in India. This delta variant is seeming to be highly contagious due to mutations in spike. Several other mutations like D614G in modulating higher spike infectivity and density, E484K for decreased antibody neutralization, N501Y and K417N for altering spike interacting with ACE receptor and antibodies derived from human were reported. [1-3]. Recent reports suggests that NTD (N-Terminal Domain) is known to be supersite for antibody mediated binding[4-6]. Reports on rigidization in NTD of spike had led to the antibody escape mechanism in this delta variant [7]. These examples are enough to show case 
how lethal this variant is in terms of transmittance, infectivity and evading host immune responses. Opposite to the same, some rare mutations like C241T was favoring host also [8] In India second wave was persisted from middle of the march 2021, till June 2021[9]. Preliminary focus of this research lies on finding possible reason of sudden drop down of second wave of SARS-CoV-2 in halved period compared to first wave with increased seroprevalence. Virus genome is extensively studied and possible mutations favoring host were identified using protein dynamics approach, among them ORF8 carrying mutations $\Delta 119$ Asp and $\Delta 120 \mathrm{Phe}$ had grabbed our attention due to their direct involvement in dimerization of ORF8 by forming hydrogen bonds and Salt-bridges. Crystal structure of ORF8 reported was taken as a reference structure for analyzing effect of these deletions using molecular modelling and simulation approach [10]. ORF8 is known to be important protein for SARS-CoV-2 mediated infection by down regulation MHC-I molecule in ER (endoplasmic reticulum pathway) mediated protein trafficking pathway [11]. ORF8 involvement in endoplasmic reticulum mediated stress and antagonizing IF-beta (interferon beta) for immune evasion is also known [12]. Deletion of ORF8 leads to decreased severity of infection as reported [13,14]. These examples show case the involvement of ORF8 in modulating host immune response and majorly by downregulating MHC-I. Exact interface of MHC-I binding with ORF8 is not known yet. In this study effect of these $\Delta 119$ Asp and $\Delta 120$ Phe deletions with respect to ORF8 dimerization is studied. Flexible induced docking was performed to study the ORF8 mediated MHC-I binding. Mutations were correlated with timeline of second wave and available cohort study on seroprevalence.

\section{Material and Methods}

\subsection{Data retrieval}

77

Crystal structure of ORF8 (PDB ID: 7JTL) protein of SARS-CoV-2 (WT_ORF8) was retrieved from protein data bank [10]. Protein sequence ORF8_GBRC_NCD_370 of SARS-CoV-2 delta variant (MUT_ORF8) was obtained from inhouse sequencing (Sequence submitted to GAISAD with accession number EPI_ISL_2001211) and fasta sequence of MHC-I protein (Accession no: NP_005505.2) was downloaded from NCBI.

\subsection{Protein structure modelling and Molecular Dynamics Simulations studies}

3-Dimentional structures of MUT_ORF8 protein as well as of MHC-I protein were built using homology modelling panel under the Schrodinger suite release 2021-2 [15]. The fasta sequences of MUT_ORF8 and MHC-I protein were imported into the Schrodinger suite. Homology blast search resulted in the templates 7JTL and 6AT5 corresponding to MUT_ORF8 and MHC-I respectively. Protein preparation wizard was then used for the refinement of protein structures. Additionally PRIME module was also used to add missing residues and pKa refinement of proteins was done using epic module of Schrodinger suite [16].

Conformational stability of WT_ORF8 and MUT_ORF8 dimers were inspected using molecular dynamic simulations studies in detail using DESMOND module implemented in Schrodinger 
suite 2021-1 till 200 nanoseconds (ns)[17]. OPLS4 force field was applied to refine the WT_ORF8 and MUT_ORF8 dimeric proteins as well as H-bonds were refilled using structure refinement panel implemented in Schrodinger suite [18,19]. Particle mesh Ewald method was applied for calculation of long-range electrostatic interactions [20]. Also, at every 1.2 ps intervals the trajectories were recorded for the analysis. The proteins WT_ORF8 and MUT_ORF8 were placed in the center of the dodecahedron water box of the TIP3P water model of size wild $353968 \AA$ and $360038 \AA$ respectively [21]. The whole system was neutralized using $1.5 \mathrm{mM}$ Salt concentration. A coupling constant of 2.0 ps under the Martyna-Tuckerman-Klein chaincoupling scheme was used for pressure control and the Nosé-Hoover chain-coupling scheme at $310.3 \mathrm{~K}$ was used for temperature control of the system [22]. The whole system was initially energy minimized by steepest descent minimization. Total negative charges on the protein structures of WT_ORF8 and MUT_ORF8 were balanced by appropriate number of Na+ ions to make the whole system neutral. Further, energy-minimized protein structures were subjected to position restrained dynamics for $200 \mathrm{~ns}$, allowing water molecules to equilibrate and the whole protein system was kept fixed. Optimized system was subjected to MD run for $200 \mathrm{~ns}$ at $310.5 \mathrm{~K}$ and 1 atmospheric pressure (NPT ensemble). The binding energy of the system was calculated for each of the protein structures and stability of complex was monitored by analyzing RMSD, RMSF, radius of gyration and $\mathrm{H}$-bonds of each dimer throughout simulation run time. High resolution images were generated using Pymol and biovia Discovery studio (BIOVIA, Dassault Systèmes, BIOVIA Workbook, Release 2020; Schrodinger, LLC. 2010. The PyMOL Molecular Graphics System). Protein networking was studied into NASP server available online [25]. Ramachandran plots were generated into zlab Ramachandran plot server [26].

\subsection{Binding energy (MMGBSA) Calculation}

115

116

117

118

119

120

121

The binding free energy of WT_ORF8 and MUT_ORF8 dimers were calculated by Prime Molecular Mechanics-Generalized Born Surface Area (MMGBSA) using thermal_mmgbsa.py implemented under PRIME module of Schrodinger suite [27-29]. The binding free energy of each protein provides a summary of the biomolecular interactions between monomeric chains of protein dimer. OPLS4 force-field and VSEB solvation model were used for MMGBSA calculation. The binding energy includes potential energy as well as polar and non-polar solvation energies were calculated as following.

$$
\Delta \mathrm{G}_{\mathrm{Bind}}=\Delta G_{\mathrm{SA}}+\Delta G_{\mathrm{Solv}}+\Delta E_{\mathrm{MM}}
$$

\subsection{Principal Component analysis (PCA) and Dynamics cross-correlation matrix (DCCM) calculation}

To perform PCA, Primarily the covariance matrix $\mathrm{C}$ was calculated. The eigenvectors and eigenvalues were obtained for the covariance matrix $\mathrm{C}$ [30]. The principal components (PCs) are projections of a trajectory on the principal modes, of which usually the first few ones are largely responsible for the most important motions. The elements $\mathrm{Cij}$ in the matrix $\mathrm{C}$ are defined as: 


$$
C_{i j}=\left\langle\left(r_{i}-\left\langle r_{i}\right\rangle\right) *\left(r_{j}-\left\langle r_{j}\right\rangle\right)\right\rangle
$$

130

131

132

133

134

135

136

137

138

139

140

141

142

143

144

145

146

147

148

149

150

151

152

153

154

155

156

157

158

159

160

161

162

163

164

From equation 1, ri and rj are the instant coordinates of the ith or jth atom, $\left\langle r_{i}\right\rangle$ and $\left\langle r_{j}\right\rangle$ and mean the average coordinate of the ith or jth atom over the ensemble.

Correlative and anti-correlative motions are playing a key role in the recognition as well as binding in the biological-complex system. These motions can be prevailed through molecular dynamics simulation trajectories by defining the covariance matrix about atomic fluctuation. The magnitude of correlative motions of two residues can be represented by the cross-correlation coefficient, Cij. It is defined by following equation:

$$
C_{i j}=\frac{\left\langle\Delta r_{i *} \Delta r_{j}\right\rangle}{\left(\left\langle\Delta r_{i}\right\rangle^{2}\left\langle\Delta r_{j}\right\rangle^{2}\right)^{1 / 2}}
$$

Here, $\mathrm{i}(\mathrm{j})$ is ith (jth) two residues (or two atoms/proteins), $\Delta \mathrm{ri}(\Delta \mathrm{rj})$ is the displacement vector corresponding to ith (jth) two residues (or two atoms/proteins), and $\langle.$.$\rangle is for the ensemble$ average. The value of $\mathrm{Cij}$ ranges from +1 to -1 . $+\mathrm{Cij}$ denotes positive correlation movement (same direction) shown in blue color, and -Cij denotes anti-correlation movement (opposite direction) shown in red color. The higher the absolute value of $\mathrm{Cij}$ is, the more correlated (or anti-correlated) the two residues (or two atoms or proteins). PCA and DCCM both were evaluated by using run trj_essential_dynamics.py, a python script under Desmond module of Schrodinger 2021-1[31].

\section{Results:}

\subsection{Effect of deletions on the binding affinity of MUT_ORF8 dimer}

WT_ORF8 protein comprises of two monomeric chains existing in the form of a dimeric structure which is tightly packed with the help of various electrostatic interactions and H-bonds (Supplementary figure S1). The key residues involved in the packing of WT_ORF8 dimers are Lys53, Arg115, Asp119, Phe120 and Ile121. Other residues involved in intra chain bonds between dimers of WT_ORF8 are Gln18, Ser24, Ala51, Arg52 and Ser54 (Figure 1A). These dimers are closely held together with four salt bridges formed between A: Asp119-B: Arg115, A: Arg115-B: Glu92, B: Asp119-A: Arg115 and B: Arg115-A: Glu92. Other interactions are several H-bonds between Phe120 and Lys53, Lys53 and Ser24, Gln18-Lue22, Arg52 and Ile121 (Figure 1A). In WT_ORF8, amino acids Asp119 and Phe120 are predominantly involved in the formation of salt bridges as well as Hydrogen bonds (Supplementary figure S1: C \& D). The detailed analysis of MUT_ORF8 dimer protein showed that its monomer is attached with each other with only 1 salt bridge between C: Arg15-D: Glu92. Six H-bonds are formed between amino acids C: Gln18-D: Ser24 (one H-bond), C: Arg115-D: Leu118 (two H-bonds) and C: Ile119-D: Ala51 (three H-bonds) (Supplementary figure S1: C \& D). Protein structural network analysis shows reduced nodes (amino acids) and bonds (edges). WT_ORF have 722 edges while Mut_ORF8 have only 714 (Figure 1B). Decreased edges correlated with reduced protein-protein 
interactions (here in case of monomers). These decreased monomeric interactions in MUT_ORF8 might leads to less stable dimer formation of ORF8. Ramachandran plot for both variants of ORF8 is shown in figure 1C. WT_ORF8 possess majority of amino acids in highly preferred region (green) with no questionable interactions, while mutant ORF8 possess two questionable angles for amino acids C: E-64 and D: S-67 (shown in red dots), depicting decrease in protein stability of MUT_ORF8 (Figure 1C). Contact plot generated for inter and intra molecular interactions within ORF8 dimers where WT_ORF8 possess a higher inter-intra molecular interactions compared to MUT_ORF8, and leads to more stable dimer (Figure 1D). Overall structural studies of proteins suggests that WT-ORF8 seems to be stable dimer compared to MUT_ORF8 by forming strong interactions like hydrogen bonds and salt bridges, these observations were further confirmed using molecular dynamics approach.

\subsection{Molecular dynamics reveals breakdown/dissociation of ORF8 dimer in delta variant}

After execution of the classical molecular dynamics simulations for 200ns the root mean square deviation (RMSD) of the trajectories were calculated, to identify the region of WT_ORF8 and MUT_ORF8 dimers showing deviations with respect to the initial structure. The RMSD plot clearly showed that the conformational stability of WT_ORF8 is greater than MUT_ORF8 (Figure 2C). The RMSD of MUT_ORF8 dimer is on higher side throughout the simulation run time as compared to initial conformations. The RMSD of WT_ORF8 has fluctuation between 1.527-5.652 $\AA$ throughout the simulation runtime of $0-200 \mathrm{~ns}$. Whereas RMSD of the MUT_ORF8 is fluctuating from $1.73 \AA$ to 4.498 during $0-10 \mathrm{~ns}, 5-10.47 \AA$ during $10-30 \mathrm{~ns}$, 10.478-12.049 ̊ during 30-100ns and 12.049-14.79 during 100-200ns. Number of H-bonds were plotted for the duration of 0-200ns simulation time (Figure 2D), showing that WT_ORF8 has number of H-bonds between 3-22 throughout the simulation. Maximum number of bonds i.e. 22 H-bonds are formed in WT_ORF8 at 102ns simulation time. Number of H-bonds were also calculated for MUT_ORF8 varying from 0 to 15. Radius of gyration was also studied to see the compactness of protein structure of WT_ORF8 and MUT_ORF8. 1119 Asp and $\Delta 120$ Phe were not favoring dimer formation in ORF8 which is seen during simulation, Supplementary video 2 shows the dissociation/breakdown of ORF8 monomers in mutant ORF8. In wild type no such breakdown occurs (See supplementary video 1) The highest radius of gyration of MUT_ORF8 throughout the simulation time, suggesting a less tight packing of MUT_ORF8 as compared to WT_ORF8 (Figure 2E). The value of radius of gyration is ranges from $18.416-24.386$ in WT_ORF8 whereas, from 18.492-25.444 in MUT_ORF8. To investigate the effect of mutation on the dynamics of the backbone atoms, RMSF values for each dimer were calculated at each time point of the trajectories. Root mean square fluctuation (RMSF) values of WT_ORF8 is shifting from 0.737 to $11.997 \AA$. Only Residues $67,68,69$ and 70 of WT_ORF8 are having high RMSF value of $11.997 \AA$, whereas other residues showing less RMSF value (Figure $2 \mathrm{H}$ ). RMSF values for MUT_ORF8 dimer is 1.3 to $7.078 \AA$. it is on higher side throughout simulation as compared to WT_ORF8. Dynamics cross-correlation matrix (DCCM) of WT_ORF8 and MUT_ORF8 were plotted (Figure 2F \& 2G) In DCCM WT_ORF8 holding higher intensity for 
blue color as compared to MUT_ORF8. Positive Cij values signaling blue colors that leads to improved interaction profile between residues.

The binding energy (MMGBSA) calculations were performed for both dimers WT_ORF8 and MUT_ORF8. From figure 3A it is clearly seen that WT_ORF8_wt is more stable having higher negative free energy as compared to MUT_ORF8. The electrostatic energy of WT_ORF8 and MUT_ORF8 was -295.08 and -97.27, respectively. Similar pattern has been observed for $\Delta \mathrm{G}$ bind, Vander Waal energy, H-bond energy, lipophilic energy, covalent energy, and solvation energy for WT_ORF8 and MUT_ORF8 (Figure 3A). It is evident that only three amino acids i.e., Arg115, Val117 and Ile121 are involved in dimerization of MUT_ORF8 as compared to WT_ORF8 where Val114, Arg115, Val116, Val117, Lue118, Asp119, Phe120 and Ile121 are involved in the stabilization of the WT_ORF8 dimer (Figure 3C). Electrostatic potential are major energies which were contributing in dimer formation. Energies were visualized in ABPS module implemented in Pymol 1.8. As shown in figure 3B, WT_ORF8 have higher opposite attraction (positive-negative) compare to MUT_ORF8. Box B1 and B2 shows the region where these electrostatic potentials persist for both variants. Increased electrostatic potential among amino-acids of WT_ORF8 shows favorable dimer formation compared to MUT_ORF8. Energy minimized dimers obtained through MMGBSA were subjected to monomer interactions. From figure 3D and 3F it is clearly depicting that WT-ORF8 have 16 combined hydrogen bonds and salt bridges while MUT_ORF8 had only 8. Minimized dimers shows about difference of 2-fold in bond formation. These results clearly indicates that Mutant ORF 8 is losing its dimer formatting capacity which might affects the virus infectivity in the host.

\subsection{Flexible docking between Variants of ORF8 and MHC-I complex}

As, the binding interface between ORF8 and MHC-I is not known yet, thus we used flexible docking to study the molecular interactions between ORF8 and MHC-I using PIPER. As shown in figure 4A, superimposed structure of docked pose of ORF8 and MHC-I were shown. Maximum possess which were generated were showing binding of ORF8 between beta macroglobulin chain and alpha 3 domain of MHC-I, where both dimers of ORF8 can easily accommodate. Pivotal interactions among WT_ORF8 with respect to MHC-I complex are 18 and MUT_ORF8 with respect to MHC-I were only 11 (Figure 4B \& 4C). Based on docking results, we hypothesized that unstable dimeric structure of ORF8 (MUT_ORF8) might not be able to bind efficiently to MHC-I complex, hence not able to capture it tightly for autophagy. These correlations further lead to enhance expression of MHC-I compared to wild-type virus infection.

\section{Discussion}

The molecular mechanism behind the severity and rapid spread of the COVID-19 disease is yet to be investigated. It is reported that ORF8 is a rapidly evolving dimeric protein that interfere with the immune responses in host [10]. There are some reports showing that ORF8 is interacting 
with proteins such as IL17RA of MHC-I molecular pathway [32]. It was also reported that SARS-CoV-2 virus infection leads to downregulation of MHC-I through direct interactions with ORF8 and selectively targeted towards lysosomal autophagy, consequently immune evasion [11]. The antigen presentation system of host will also be impaired due to ORF8-MHC-I interactions. So ORF8 has now become a prime target for scientist to investigate the mechanism behind ORF8-MHC-I interactions. During second wave of COVID-19 disease, although the infection rate was very high, it was seen that hosts developed adaptability towards the COVID19 infection. Therefore, the study was planned with two objectives firstly, exhaustive analysis of the molecular structures of ORF8 dimer of wild type and delta variant (WT_ORF8 and MUT_ORF8) and secondly the interactions between WT_ORF8-MHC-I complex and MUT_ORF8-MHC-I complex. The detailed analysis of dimeric structures of WT_ORF8 and MUT_ORF8 showed a significant difference in interaction pattern between monomeric chain. In WT_ORF8 the key interaction is formed between Asp119 and Phe120 (Figure 1C). Whereas, due to deletion of Asp119 and Phe120 amino acids in MUT_ORF8 the interactions between MUT_ORF8 monomeric chains were diluted (Figure 1A). Deletion of Asp119 and Phe120 in MUT_ORF8 protein of SARS CoV2 delta variant caused loss of three salt bridges as well as Hbonds. The structural instability of the MUT_ORF8 can be clearly witnessed through molecular dynamics simulation studies. In MD studies RMSD, RMSF and radius of gyration of MUT_ORF8 is always towards higher side as compared to WT_ORF8 (Figure 2C, 2D, \& 2F). It was also observed at many time points of simulation the number of hydrogen bonds tends to zero in MUT_ORF8 indicating that there was loss of connectivity between the monomeric chains of MUT_ORF8 (Figure 3D). But in WT_ORF8 there are constant interactions between the monomeric chains reveling the conformational stability of the dimeric structure. Higher RMSF values for MUT_ORF8 dimer throughout simulation indicates the greater flexibility. Additionally, the radius of gyration was also calculated for ORF8_WT and MUT_ORF8 dimers to study the compactness of these dimeric structure with protein folding and unfolding over thermodynamic principals during the 200ns of the molecular dynamics simulation. It is evident that only three amino acids i.e., In MUT_ORF8, amino acids Arg115, Ile119, Ala51, Ser24 are involved in bond formation between the dimers, whereas Phe120 and Lys53, Lys53 and Ser24, Gln18-Lue22, Arg52 and Ile121 in addition to A: Asp119-B: Arg115, A: ARG115-B: Glu92, B: Asp119-A: Arg115 and B: Arg115-A: Glu92 are involved in the stabilization of the WT_ORF8. Interestingly, in addition to these interaction two pi-Sulphur bonds were also observed between A: Phe120-B: Cys90 and A: Phe120-B: Cys25 in WT_ORF8, which is totally absent in MUT_ORF8 due to deletion of Phe120 amino acid. As Ala51 and Ser24 are major interacting amino acid in case of MUT_ORF8 its detail interaction map was built that surprisingly showed that it is these two amino acids are forming unfavorable bonds i.e. D: Ala51-D: Ser97 and C: Ser24-D: Lys53, which is also contributing towards instability of MUT_ORF8.

The stability of ORF8 dimers seems to be one of the major reasons contributing towards the host accommodate on the surface of MHC-I complex, whereas MUT_ORF8 is unable to firmly 
accommodate on the surface of MHC-I complex causing escape of MHC-I complex towards lysosomal autophagy and contributing consequently in increased immune response.

Nationwide population weighted study of seroprevalence from May-June 2020 was conducted by ICMR (Indian council of medical research), showing $0.75 \%$ among 21 states [33]. While in second seroprevalence study using Abbott assay detecting IGg antibodies against SARS-CoV-2 nucleoprotein, in August 2020 showed increased seroprevalence to 6.6\% (95\% CI 5.8-7.4) [34]. Seroprevalence among adults increased by about ten times, from $0.7 \%$ in May, 2020, to $7 \cdot 1 \%$ in August, 2020 in India [35]. Supplementary figure 3A, showing number of SARS-CoV-2 cases reported in India during first and second wave. Third seroprevalence data shows percentage increase to $24.1 \%$ from December 2020 to January 2021 (Supplementary figure 3B). Drop down of $50 \%$ cases SARS-CoV-2 cases during second wave was double quick time compared to first wave. During first wave in $17^{\text {th }}$ September 2020 cases were 93735 and cases were halved in 6 weeks, $30^{\text {th }}$ October 2020 with 46380 cases (Supplementary figure 3C). While in second wave higher number of cases $(3,91,261)$ where decreased $(1,95,183)$ in half time compared to first wave. ICMR $4^{\text {th }}$ seroprevalence data shows $70 \%$ of Indian population (unvaccinated) showing IgG antibody titer against SARS-CoV-2 cases (ICMR $5^{\text {th }}$ Seroprevalece data). Drastic increase in seroprevalence after second wave, from $0.75 \%$ to $70 \%$ is unusual observation for high transmittable delta variant. Delta variant have these D119, F120 deletions, which were disrupting ORF8, responsible for downregulating MHC-I and suppressing host immune response. Results of the study suggests that the dimerization of MUT_ORF8 is altered, that might be affecting the ORF8 mediated MHC-I downregulation by autophagy in delta variant.

Second in India was not only due to predominant delta variant but other lineages were also involved. In such cases strong case study or proof is required in support of this hypothesis that antibody response was due to loss of dimerization capacity of ORF8. In nationwide study seroprevalence was detected for all kind of SARS-CoV-2 lineage infections, but here we are studying only delta mediated immune responses. To support this nationwide study, State wise seroprevalence was also studied in region like Ahmedabad, Gujarat having higher number of active cases of SARS-CoV-2 infection. Supplementary figure shows the genome sequencing data of Gujarat biotechnology research center during second wave, where B.1.167.2 (red) lineage (delta) was found to be $100 \%$ in samples collected from patients [37]. $5^{\text {th }}$ seroprevalence data of Ahmedabad city is shown in Supplementary figure S4B. Seroprevalence due to delta only was 81.93\% (Ahemdabad Summary, 2021). We had hypothesized that altered dimer of ORF8 might not able to perform autophagy of MHC-I molecule compared to wild-type ORF8, which might lead to favoring host immune responses. This can be one possible reason for the sudden drop down of cases during second wave in India.

\section{Conclusion}


317 Frequency of delta variant during second wave in India was persisted 9.6-76.5\% in India while in 318 Gujarat it was between 18.96 to $90 \%$ (Figure 5D). $5^{\text {th }}$ seroprevalence study by ICMR shows $31962.3 \%$ population have antibodies due to virus infection, while in Gujarat there $81.93 \%$ 320 seroprevalence was observed. These patterns leads to conclude that as the frequency of delta is 321 increasing seroprevalence among population had also increased (Figure 5C \& 5D). These 322 seroprevalence study supports our hypothesis that loss in dimerization capacity of ORF8 (from

323 delta variant) leads to an abrogation of ORF8 MHC-I interaction and overcome suppression of 324 adaptive immune response.

\section{Conflict of Interest}

326 The authors declare that the research was conducted in the absence of any commercial or financial 327 relationships that could be construed as a potential conflict of interest.

\section{Authors contributions}

329

AC and IS performed Insilco experiments, Molecular dynamics, validated hypothesis and wrote the manuscript. AP, MJ, and CJ provided funding, validated results, and corrected manuscript.

\section{Data Availability Statement}

332 Seroprevalence study were performed based on ICMR (Indian council of medical research), PIB 333 (Press information bureau), Times of India (TOI), Gujarat Biotechnology research center's 334 COVID19 portal (GBRC), MoHFW (Ministry of health and family welfare), Nextstrain and 335 GISAID data base.

ICMR: https://www.icmr.gov.in/

337 PIB: https://pib.gov.in/PressReleseDetail.aspx?PRID=1748351

TOI: https://timesofindia.indiatimes.com/

339 GBRC: https://covid.gbrc.res.in/

MoHFW: https://www.mohfw.gov.in/

Nextstrain: https://nextstrain.org/ncov/gisaid/global

GISAID: https://www.gisaid.org/index.php?id=209

344 Funding is provided by Department of Science and Technology (DST) India.

\section{Figures}


A

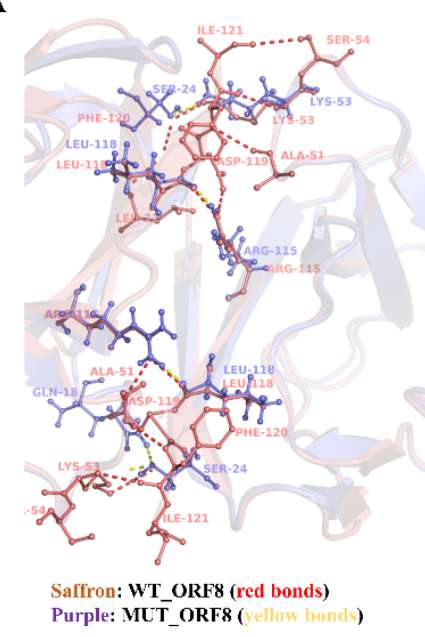

B
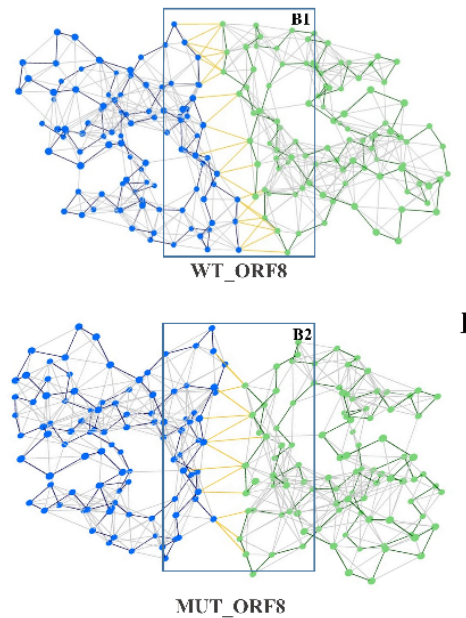

C

D
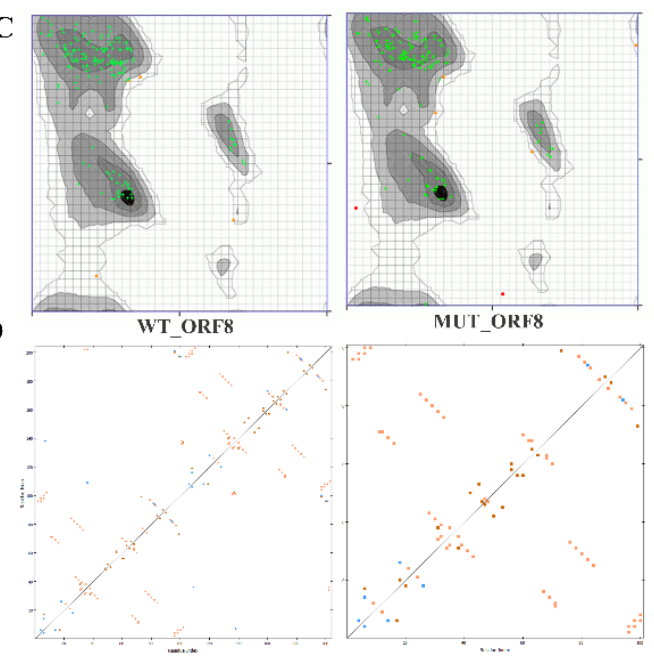

Figure 1: Change in bond formation within WT_ORF8 and MUT_ORF8 due to 119Asp and 120F deletion: 1A: Superimposition of WT_ORF8 shown in saffron color and MUT_ORF8 shown in purple color. Hydrogen bond formation within two monomeric units of ORF8 is illustrated using Pymol where red and yellow bonds representing bond formation within WT_ORF8 and MUT_ORF8. 1B: Network analysis of protein structures using NASP sever, where B1 and B2 represent network between dots as a node (amino acids) and inter and intramolecular bonds as an edge (yellow) for WT_ORF8 and MUT_ORF8 respectively. WT_ORF8 possess 203 nodes and 722 edges while MUT_ORF8 possess 202 nodes and 714 edges. 1C: Ramachandran plot for WT_ORF8 and MUT_ORF8. Green dots represent highly preferred observations, yellow dots represent preferred observations and red dots represents questionable observations. MUT_ORF8 possess two questionable observations which are C: E64 and D: S-67, while WT_ORF8 posses no such kind of observations. 1D: Contact plot showing amino-acids contacts between monomeric units of WT_ORF8 and MUT_ORF8. Blue color shows main chain-side chain interactions, Saffron color shows main chain-main chain interactions, and brown color shows side chain-side chain interactions within monomeric subunits. 
$\mathbf{A}$

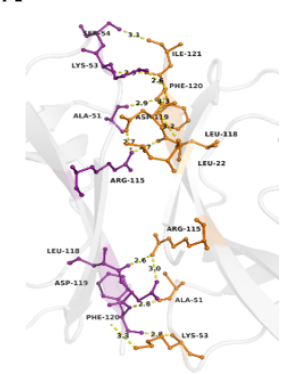

B

Mut_ORF8

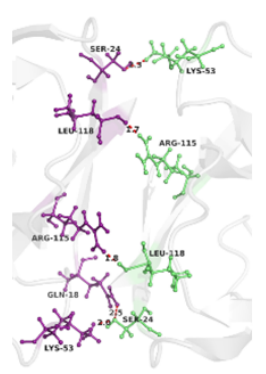

D
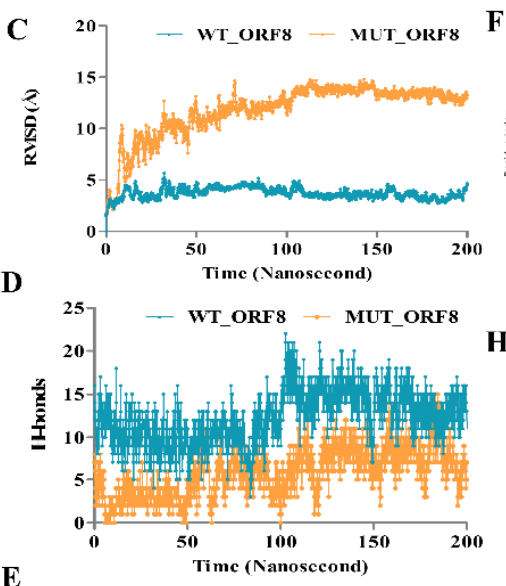

$\mathbf{E}$

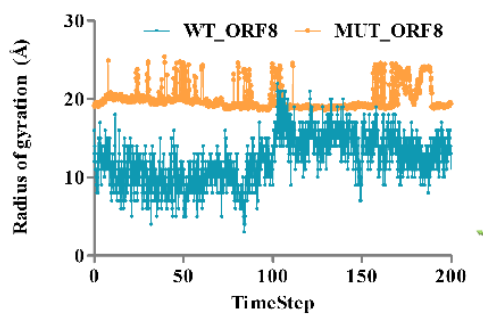

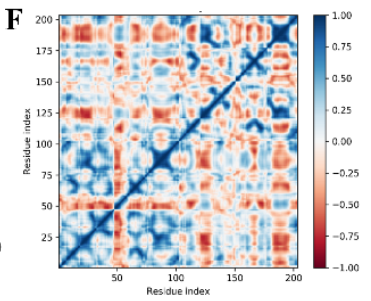

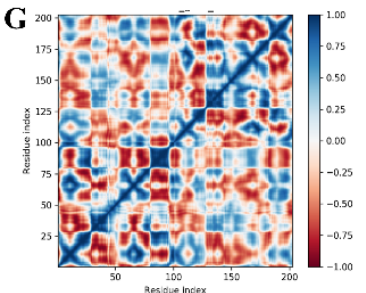

H
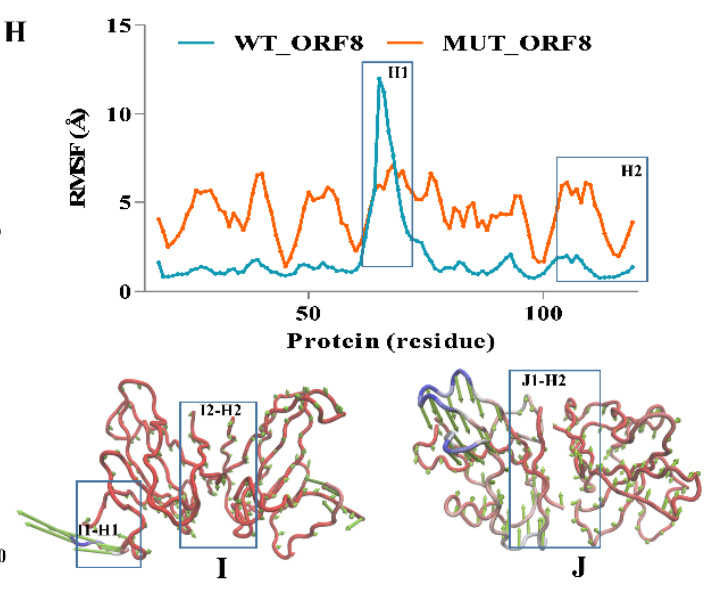

Figure 2: Molecular dynamics studies for both variants of ORF8 dimer. 2A: Intramolecular interactions between WT_ORF8 monomeric subunits 2B: Intramolecular interactions between MUT_ORF8 monomeric subunits. 2C: RMSD (root mean square deviation) within WTORF8(cyan) and MUT_ORF8 (orange) complex. 2D: Hydrogen bonds formation within WTORF8(cyan) and MUT_ORF8 (orange) complex. 2D: Radius of gyration for WT-ORF8(cyan) and MUT_ORF8 (orange) complex 2F \& 2G: Dynamics cross-correlation matrix obtained from trajectories of WT_ORF8 and MUT_ORF8 complexes respectively. Blue to red color represents the cij values between 1 to -1 . No cross correlation was shown by white color. 2H: RMSF (root mean square fluctuation) in WT-ORF8(cyan) and MUT_ORF8 (orange) complex. 2I: PCA1 mode of WT-ORF8, length of arrow is in linear relation between protein dynamics/fluctuation during trajectories blue color shows highly dynamic regions, while red color shows less dynamics regions. 2J: PCA1 mode of MUT-ORF8. 
A

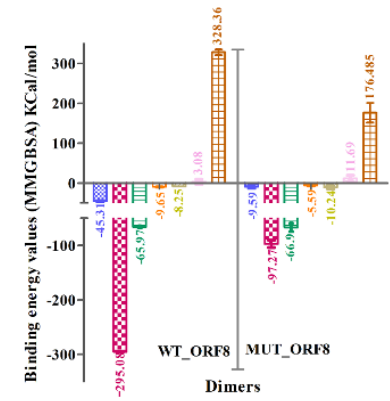

B

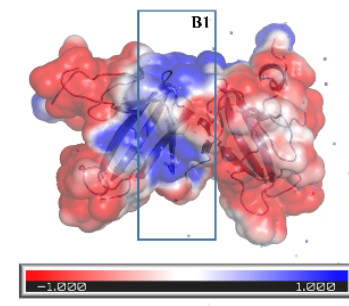

WT_ORF8

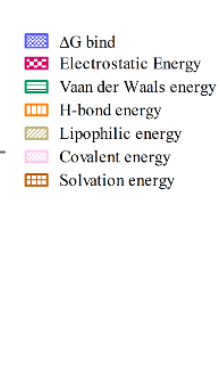

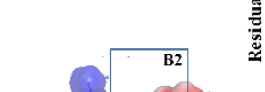

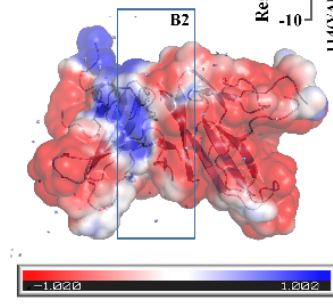

MUT_ORF8

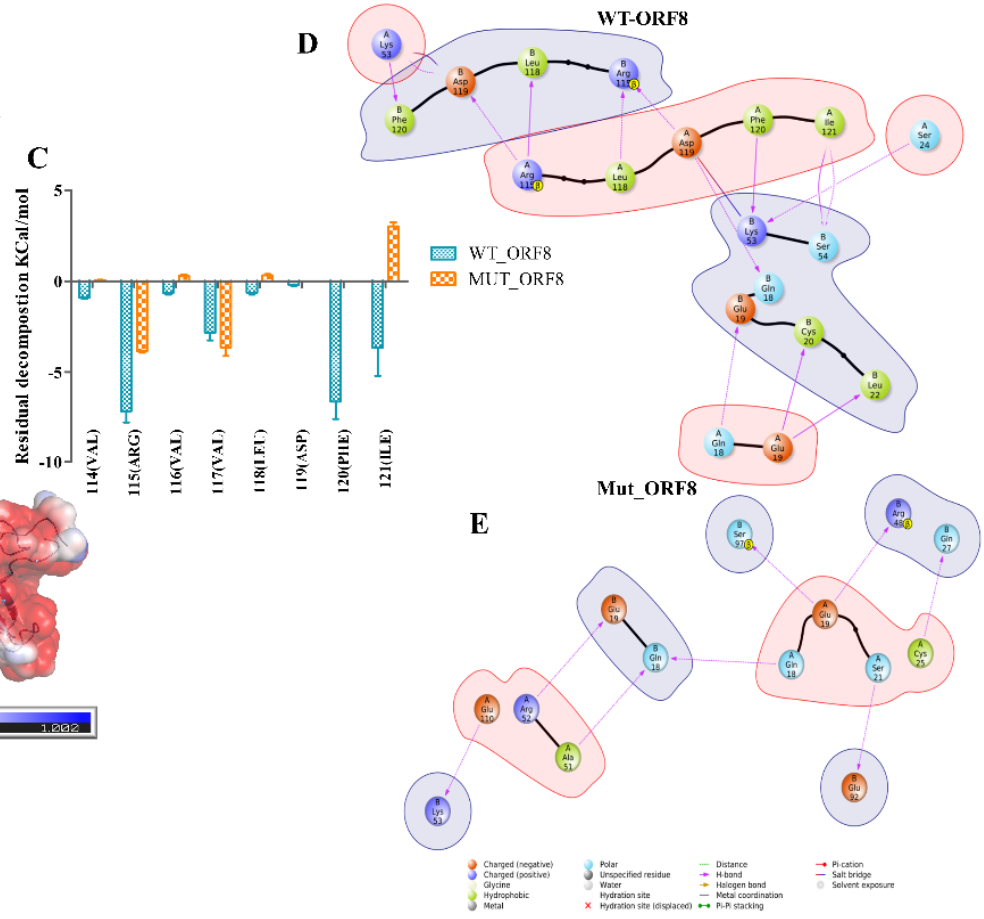

377 Figure 3: Binding energy studies within dimers of ORF8. 3A: Binding energy difference 378 between WT_ORF8 and MUT_ORF8. Major energies involved in dimer formation are shown in 379 different legends. 3B: electrostatic interaction map drawn for $1^{\text {st }}$ energy minimized dimer 380 obtained from MMGBSA approach. Blue, white and red colors represent positive, null, negative 381 electrostatic potential respectively, inform of surface representations. B1 \& B2 represents 382 potential between two monomeric subunits of WT-ORF8 and MUT-ORF8 respectively. 3C: 383 Thermal decomposition among amino-acids residues within both dimers. WT_ORF8 (cyan) and 384 MUT_ORF8 (Orange) showing decomposition energies for key residues involved in dimer formations. 3D \& 3E: Interactions among energy minimized dimers obtained through MMGBSA, legends for each type of bond is shown in under figure $3 \mathrm{E}$. 
B

A

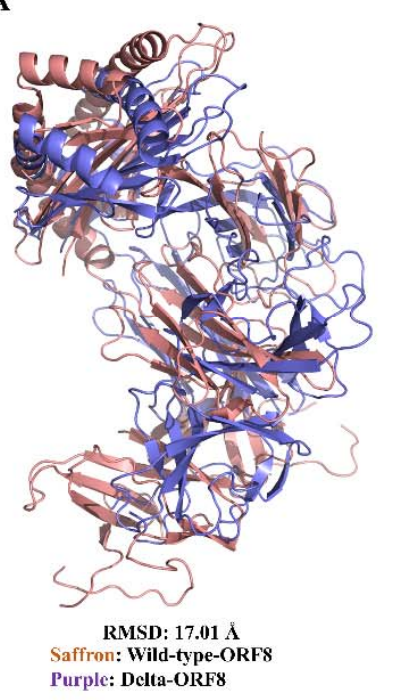

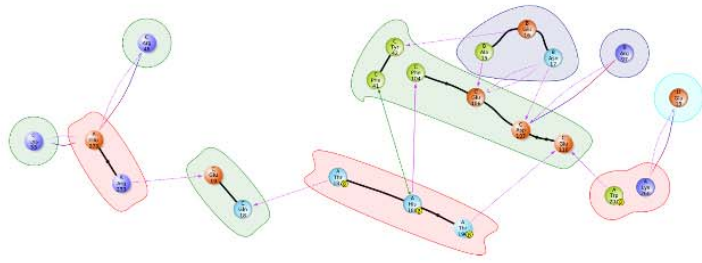

C
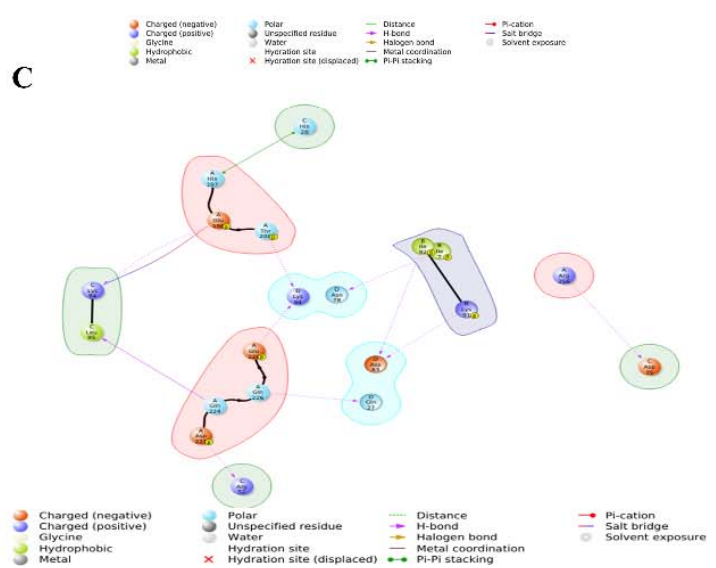

388 Figure 4: Flexible docking of MHC-I with ORF8 dimer. A: Superimposed structure of 389 WT_ORF8_MHC-I(saffron) and MUT_ORF8_MHC-I (purple). B: Pivotal interaction among 390 WT_ORF8_MHC-I complex. C: Pivotal interaction among MUT_ORF8_MHC-I complex. 
A

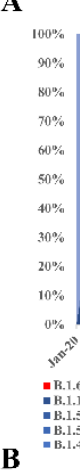
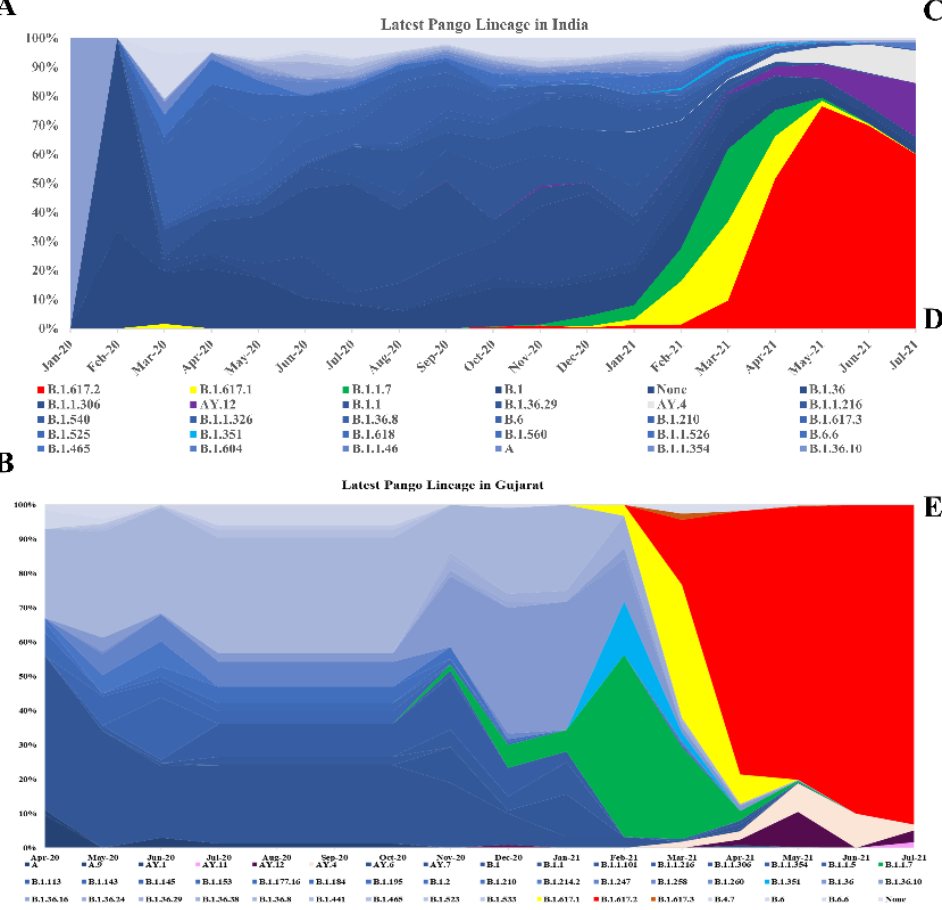

C

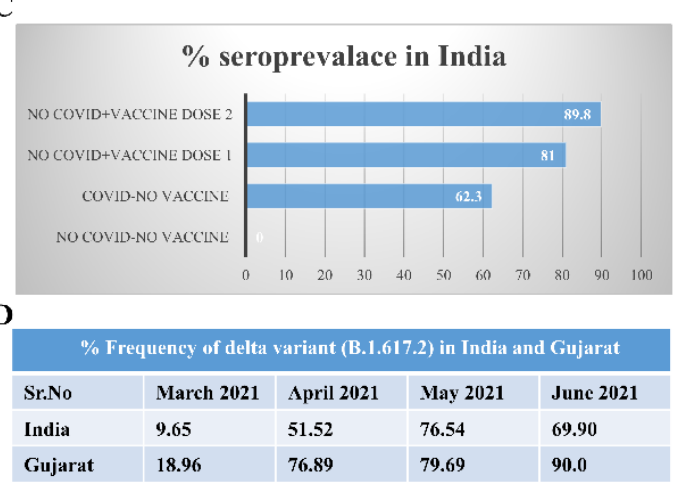

$\mathbf{E}$

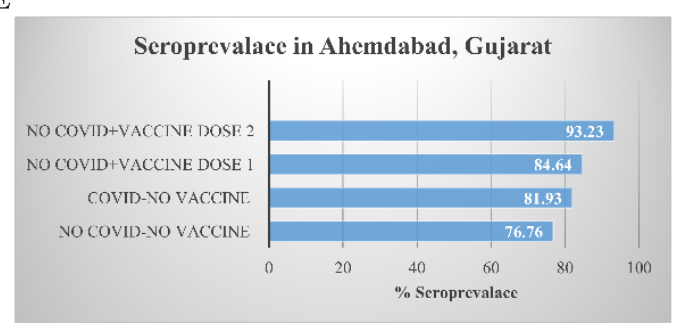

Figure 5: Nationwide and statewide seroprevalence study: 5A: SARS-CoV-2 sequences submitted to GAISAD database from India at different time scale with latest Pango lineage. 5B: SARS-CoV-2 sequences submitted to GAISAD database from Gujarat at different time scale with latest Pango lineage. 5C: $5^{\text {th }}$ Seroprevalence data from ICMR (Indian council of medical research). 5D: Table narrating frequency of delta variant (B.1.617.2) during second wave in India and Gujarat. 5E: $5^{\text {th }}$ Seroprevalence data FROM Ahmedabad city, Gujarat. 


\section{References}

411

412

413

414

415

416

417

418

419

420

421

422

423

424

425

426

427

428

429

430

431

432

433

434

435

436

437

438

439

440

441

442

443

444

445

[1] Fratev F. The SARS-CoV-2 S1 spike protein mutation N501Y alters the protein interactions with both hACE2 and human derived antibody: A Free energy of perturbation study. BioRxiv 2020:2020.12.23.424283. https://doi.org/10.1101/2020.12.23.424283.

[2] Zhang L, Jackson CB, Mou H, Ojha A, Peng H, Quinlan BD, et al. SARS-CoV-2 spikeprotein D614G mutation increases virion spike density and infectivity. Nature Communications 2020;11:1-9. https://doi.org/10.1038/s41467-020-19808-4.

[3] Jangra S, Ye C, Rathnasinghe R, Stadlbauer D, Alshammary H, Amoako AA, et al. SARS-CoV-2 spike E484K mutation reduces antibody neutralisation. The Lancet Microbe 2021:1-2. https://doi.org/10.1016/S2666-5247(21)00068-9.

[4] McCallum M, De Marco A, Lempp FA, Tortorici MA, Pinto D, Walls AC, et al. Nterminal domain antigenic mapping reveals a site of vulnerability for SARS-CoV-2. Cell 2021;184:2332-2347.e16. https://doi.org/10.1016/j.cell.2021.03.028.

[5] Chi X, Yan R, Zhang J, Zhang G, Zhang Y, Hao M, et al. A neutralizing human antibody binds to the N-terminal domain of the Spike protein of SARS-CoV-2. Science 2020;369:650-5. https://doi.org/10.1126/science.abc6952.

[6] Soh WT, Liu Y, Nakayama EE, Ono C, Torii S, Nakagami H, et al. The N-terminal domain of spike glycoprotein mediates SARS-CoV-2 infection by associating with LSIGN and DC-SIGN. BioRxiv 2020:1-30.

[7] Chaudhari AM, Kumar D, Joshi M, Patel A, Joshi C. E156G and Arg158, Phe-157/del mutation in NTD of spike protein in B.1.617.2 lineage of SARS-CoV-2 leads to immune evasion through antibody escape. BioRxiv 2021.

https://doi.org/10.1101/2021.06.07.447321.

[8] Chaudhari A, Chaudhari M, Mahera S, Saiyed Z, Nathani NM, Shukla S, et al. In-Silico analysis reveals lower transcription efficiency of C241T variant of SARS-CoV-2 with host replication factors MADP1 and hnRNP-1. Informatics in Medicine Unlocked 2021:100670. https://doi.org/https://doi.org/10.1016/j.imu.2021.100670.

[9] Wordometer. No Title. Worldometer COVID-19 Coronavirus Pandemic 2021; Published Online August 2021.

[10] Flower TG, Buffalo CZ, Hooy RM, Allaire M, Ren X, Hurley JH. Structure of SARS-cov2 ORF8, a rapidly evolving immune evasion protein. Proceedings of the National Academy of Sciences of the United States of America 2021;118:1-6. https://doi.org/10.1073/pnas.2021785118.

[11] Zhang Y, Zhang J, Chen Y, Luo B, Yuan Y, Huang F, et al. The ORF8 protein of SARSCoV-2 mediates immune evasion through potently downregulating MHC-I. BioRxiv 2020. https://doi.org/10.1101/2020.05.24.111823. 
[12] Rashid F, Dzakah EE, Wang H, Tang S. The ORF8 protein of SARS-CoV-2 induced endoplasmic reticulum stress and mediated immune evasion by antagonizing production of interferon beta. Virus Research 2021;296:198350. https://doi.org/10.1016/j.virusres.2021.198350.

[13] Zinzula L. Lost in deletion: The enigmatic ORF8 protein of SARS-CoV-2. Biochemical and Biophysical Research Communications 2021;538:116-24. https://doi.org/10.1016/j.bbrc.2020.10.045.

[14] Pereira F. SARS-CoV-2 variants combining spike mutations and the absence of ORF8 may be more transmissible and require close monitoring. Biochemical and Biophysical Research Communications 2021;550:8-14. https://doi.org/10.1016/j.bbrc.2021.02.080.

[15] Madhavi Sastry G, Adzhigirey M, Day T, Annabhimoju R, Sherman W. Protein and ligand preparation: parameters, protocols, and influence on virtual screening enrichments. Journal of Computer-Aided Molecular Design 2013;27:221-34. https://doi.org/10.1007/s10822-013-9644-8.

[16] Shelley JC, Cholleti A, Frye LL, Greenwood JR, Timlin MR, Uchimaya M. Epik: a software program for pKaprediction and protonation state generation for drug-like molecules. Journal of Computer-Aided Molecular Design 2007;21:681-91. https://doi.org/10.1007/s10822-007-9133-z.

[17] SC '06: Proceedings of the 2006 ACM/IEEE Conference on Supercomputing, New York, NY, USA: Association for Computing Machinery; 2006.

[18] Steinbrecher T, Abel R, Clark A, Friesner R. Free Energy Perturbation Calculations of the Thermodynamics of Protein Side-Chain Mutations. Journal of Molecular Biology 2017;429:923-9. https://doi.org/https://doi.org/10.1016/j.jmb.2017.03.002.

[19] van Zundert GCP, Moriarty NW, Sobolev O V, Adams PD, Borrelli KW. Macromolecular refinement of X-ray and cryoelectron microscopy structures with Phenix/OPLS3e for improved structure and ligand quality. Structure 2021. https://doi.org/10.1016/j.str.2021.03.011.

[20] Toukmaji AY, Board JA. Ewald summation techniques in perspective: A survey. Computer Physics Communications 1996;95:73-92. https://doi.org/10.1016/00104655(96)00016-1.

[21] Zielkiewicz J. Structural properties of water: Comparison of the SPC, SPCE, TIP4P, and TIP5P models of water. Journal of Chemical Physics 2005;123. https://doi.org/10.1063/1.2018637.

[22] Martyna GJ, Klein ML, Tuckerman M. Nosé-Hoover chains: The canonical ensemble via continuous dynamics. The Journal of Chemical Physics 1992;97:2635-43. https://doi.org/10.1063/1.463940.

[23] Schrodinger, LLC. 2010. The PyMOL Molecular Graphics System V 1. pymol n.d. 
[24] BIOVIA, Dassault Systèmes, BIOVIA Workbook, Release 2020; BIOVIA Pipeline Pilot, Release 2020 SDDS. No Title n.d.

[25] Chakrabarty B, Parekh N. NAPS: Network analysis of protein structures. Nucleic Acids Research 2016;44:W375-82. https://doi.org/10.1093/nar/gkw383.

[26] Anderson RJ, Weng Z, Campbell RK, Jiang X. Main-chain conformational tendencies of amino acids. Proteins: Structure, Function and Genetics 2005;60:679-89. https://doi.org/10.1002/prot.20530.

[27] Lyne PD, Lamb ML, Saeh JC. Accurate prediction of the relative potencies of members of a series of kinase inhibitors using molecular docking and MM-GBSA scoring. Journal of Medicinal Chemistry 2006;49:4805-8. https://doi.org/10.1021/jm060522a.

[28] Greenidge PA, Kramer C, Mozziconacci JC, Wolf RM. MM/GBSA binding energy prediction on the PDBbind data set: Successes, failures, and directions for further improvement. Journal of Chemical Information and Modeling 2013;53:201-9. https://doi.org/10.1021/ci300425v.

[29] Beard H, Cholleti A, Pearlman D, Sherman W, Loving KA. Applying Physics-Based Scoring to Calculate Free Energies of Binding for Single Amino Acid Mutations in Protein-Protein Complexes 2013;8:1-11. https://doi.org/10.1371/journal.pone.0082849.

[30] Kormos BL, Baranger AM, Beveridge DL. A study of collective atomic fluctuations and cooperativity in the U1A-RNA complex based on molecular dynamics simulations. Journal of Structural Biology 2007;157:500-13. https://doi.org/10.1016/j.jsb.2006.10.022.

[31] Chang S, Hu J, Lin P, Jiao X, Tian X. Substrate recognition and transport behavior analyses of amino acid antiporter with coarse-grained models. Molecular BioSystems 2010;6:2430-2438. https://doi.org/10.1039/c005266c.

[32] Lin X, Fu B, Yin S, Li Z, Liu H, Zhang H, et al. ORF8 contributes to cytokine storm during SARS-CoV-2 infection by activating IL-17 pathway. IScience 2021;24:102293. https://doi.org/10.1016/j.isci.2021.102293.

[33] Res M, Ruts C, Hospital CR, Sciences M, Committee IE, Crh-smims S. Prevalence of 2018:517-20. https://doi.org/10.4103/ijmr.IJMR.

[34] John J, Kang G. Tracking SARS-CoV-2 infection in India with serology. The Lancet Global Health 2021;9:e219-20. https://doi.org/10.1016/S2214-109X(20)30546-5.

[35] Murhekar M V., Bhatnagar T, Selvaraju S, Saravanakumar V, Thangaraj JWV, Shah N, et al. SARS-CoV-2 antibody seroprevalence in India, August-September, 2020: findings from the second nationwide household serosurvey. The Lancet Global Health 2021;9:e257-66. https://doi.org/10.1016/S2214-109X(20)30544-1.

[36] Research ICOM. https://pib.gov.in/PressReleasePage.aspx?PRID=1739902 2021. 
518 [37] Gujarat Biotechnology Research center, Gandhinagar G. covid.gbrc.res.in 2021.

519 [38] Summary E. 5 th Serosurveillance study in Ahmedabad EXECUTIVE SUMMARY

520 TITLE $\square$ : ASSESSING POPULATION BASED SEROPOSITIVITY FOR ANTIBODIES

521 $\operatorname{Aim} \square$ : Objectives $\square: 2021$. 